Research Paper

\title{
Multidisciplinary team consultation for resectable Gastric Cancer: A propensity score matching analysis
}

\author{
Yonghe Chen ${ }^{1,6^{*}}$, Jun Xiang ${ }^{1,6^{*}}$, Dan Liu ${ }^{2 *}$, Jian Xiao ${ }^{3,6}$, Fei Xiong4,6, Kaikai Wei ${ }^{4,6}$, Aihong Liu ${ }^{1,6}$, Shi Chen ${ }^{1,6}$, \\ Yaxi Zhu ${ }^{5,6}$, Xiaochun Meng ${ }^{4,6}$ and Junsheng Peng ${ }^{1,6 \bowtie}$ \\ 1. Department of Gastrointestinal Surgery, The Sixth Affiliated Hospital, Sun Yat-sen University, Guangzhou, 510655, China. \\ 2. Department of Laboratory Science, The Second Affiliated Hospital, Guangzhou University of Chinese Medicine, Guangzhou, 510105, China. \\ 3. Department of Medical Oncology, The Sixth Affiliated Hospital, Sun Yat-sen University, Guangzhou, 510655, China. \\ 4. Department of Radiology, The Sixth Affiliated Hospital, Sun Yat-sen University, Guangzhou, 510655, China \\ 5. Department of Pathology, The Sixth Affiliated Hospital, Sun Yat-sen University, Guangzhou, 510655, China. \\ 6. Guangdong Institute of Gastroenterology, Guangdong Provincial Key Laboratory of Colorectal and Pelvic Floor Diseases, Guangzhou, 510655, China. \\ *These authors contributed equally to this work.
}

$\square$ Corresponding authors: Dr. Xiaochun Meng (E-mail: mengxch3@mail.sysu.edu.cn), Professor, Chief, Department of Radiology; Dr. Junsheng Peng (E-mail: pengjsh@mail.sysu.edu.cn), Professor, Chief, Department of Gastrointestinal Surgery, The Sixth Affiliated Hospital, Sun Yat-sen University, 26 Yuancun Erheng Road, Guangzhou, 510655, China.

(1) The author(s). This is an open access article distributed under the terms of the Creative Commons Attribution License (https://creativecommons.org/licenses/by/4.0/). See http://ivyspring.com/terms for full terms and conditions.

Received: 2020.09.16; Accepted: 2021.01.06; Published: 2021.01.30

\begin{abstract}
Purpose: Previous studies proposed that the multidisciplinary team (MDT) consultation could improve tumor staging accuracy and outcomes of patients with gastric malignancy. However, evidence-based reports remain limited. This study aimed to determine the effectiveness of MDT for tumor staging accuracy and outcomes of patients with resectable gastric cancer, and to explore the potential factors affecting its effectiveness.

Methods: This retrospective study enrolled 719 gastric cancer patients who underwent gastrectomy in our hospital. After propensity score matching, 378 patients were selected, including 189 in the non-MDT group and 189 in the MDT group. Data regarding baseline characteristics, staging, treatments, and survival were analyzed.

Results: The data showed that the staging accuracy in the MDT group and non-MDT group was comparable ( $53 \%$ vs $61 \%$ for T stage, $46.1 \%$ vs $35.3 \%$ for $\mathrm{N}$ stage, and $78.3 \%$ vs $78.7 \%$ for $M$ stage). The MDT group had a higher proportion of preoperative chemotherapy $(39.2 \%$ vs $28 \%, p=0.03)$ and laparoscopic surgery $(82.5 \%$ vs $72 \%, p=0.02)$ than the non-MDT group. However, the achievement of RO resection was similar in the two groups $(93.7 \%$ vs $88.9 \%, p=0.73)$. There was no significant difference in the 1-year and 3-year overall survival rates between the two groups. Moreover, we observed poor patient compliance when the MDT recommended further examinations, radiotherapy, or chemotherapy before surgical interventions.

Conclusion: MDT consultation has limited effects on improving the staging accuracy and treatment outcomes including survival of patients with resectable gastric cancer. Poor patient compliance may be a factor affecting the effectiveness of MDT consultation.
\end{abstract}

Key words: multidisciplinary team consultation; gastric cancer; propensity score matching; survival; patient compliance

\section{Introduction}

Gastric cancer is the fifth most common malignancy in the world and the third leading cause of cancer-related deaths [1]. Surgical resection is the main approach for the management of resectable gastric cancer. However, preoperative management (such as accurate staging, pathological assessment, chemotherapy intervention, and radiotherapy) is also critical for improving patient outcomes. There are still controversies about the treatment options and management of patients at different stages; and the 
recommended treatment plan varies from different guidelines $[2,3]$. Therefore, there has been a growing demand in multidisciplinary teams (MDT) integrating strategies of multiple disciplines, to ensure the treatment plans to be thorough, standardized, and effective [4].

The MDT approach can be broadly defined as an integrated team effort that aims to develop individualized treatment plans for patients through improved communication, coordination, and decision making between health care professionals $[5,6]$. Although it has been widely accepted as a "gold standard" in the care of cancer patients, current literature suggests that the quality and the effectiveness of the MDT approach could be varied among different types of cancers and different centers [6]. Moreover, many factors derived from both healthcare providers and cancer patients may affect how well the MDT is implemented in the care of cancer patients; and efforts remained to be made to address these factors.

Previous studies proposed that MDT could improve the accuracy of tumor location and stage and could be beneficial to patient survival [7-9]. However, these studies mainly focus on the methodology about organizing MDT management, and studies with detailed data or strong evidence demonstrating the impact of MDT consultation on treatment outcomes of cancer patients are still limited.

In this retrospective study, we analyzed the data of patients with resectable gastric cancer who underwent gastrectomy in the past 5 years, and compared the data between patients with and without MDT consultation before surgery, with the aim to determine the effect of MDT on tumor staging accuracy and treatment outcomes and to identify factors affecting its effectiveness.

\section{Methods}

\section{Study population and data collection}

The patients' recruitment process is shown in Figure 1. During the initial screening process, we identified 733 patients who underwent gastrectomy from the gastric cancer database of The Sixth Affiliated Hospital of Sun Yat-sen University from February 2014 to August 2019. Among them, 14 patients with insufficient data were excluded, leaving 719 patients, of which 206 received MDT consultation before surgery and 513 didn't. Their demographic information including gender, age, body mass index (BMI), tumor location, differentiation, clinical stage, MDT recommendation, surgical outcomes, pathological stage, and survival were collected. This study was reviewed and approved by the Ethics
Committee of The Sixth Affiliated Hospital, Sun Yat-Sen University. This study was conducted in accordance with the 1964 Helsinki Declaration. All study participants, or their legal guardians, were provided with informed consent prior to study by the follow-up office.

\section{Multidisciplinary team consultation clinical model}

The Multidisciplinary expert panel for gastric cancer at The Sixth Affiliated Hospital of Sun Yat-sen University consisted of gastroenterological surgeons, medical oncologists, radiation oncologists, radiologists, pathologists, and a coordinator. It was a weekly clinic open every Wednesday of the week. Gastric cancer patients with complicated conditions would be recommended to the MDT clinic by the doctor in charge. Generally, we required that the patients should have a confirmed diagnosis of gastric cancer with histological evidence and had complete necessary examination such as endoscopic inspection, computed tomography scan of chest, abdomen, and pelvis. On the consulting conference, the patient's history, along with the imaging and pathological slide, would be presented and reassessed by a senior radiologist and pathologist, to confirm the diagnosis and adjust the clinical stage according to the American Joint Committee on Cancer staging criteria [10].

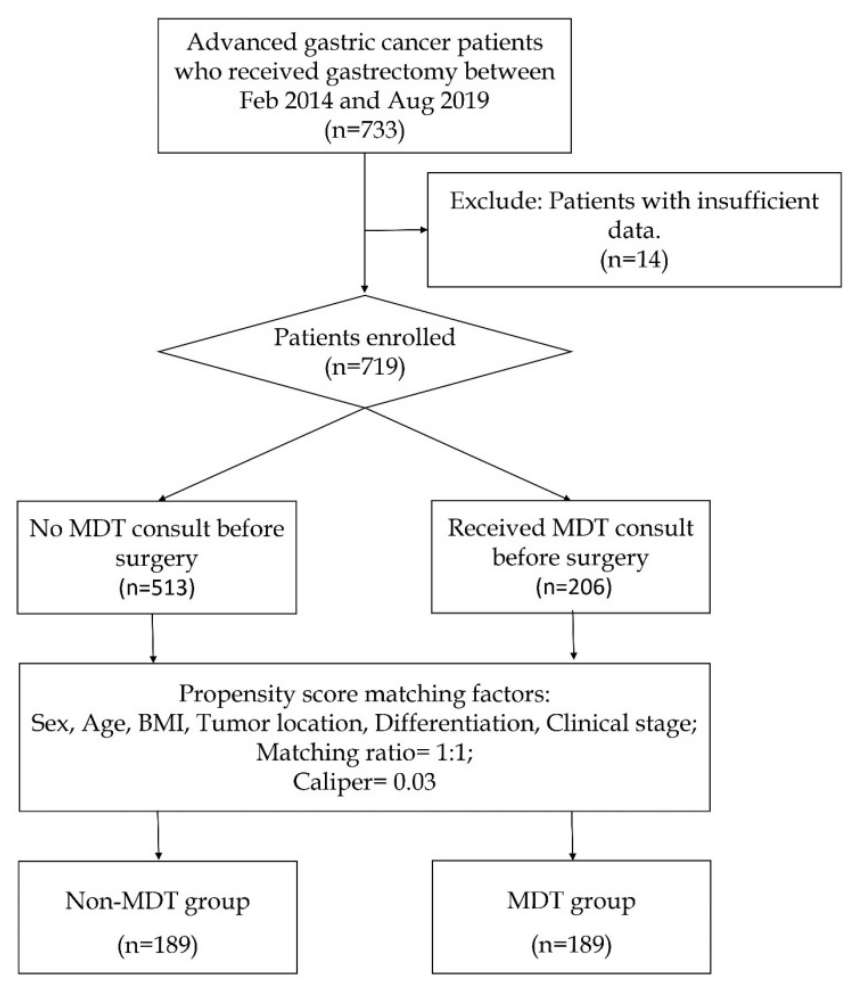

Figure 1. The flowchart showing the process of patient enrollment and propensity score matching. 
If the $\mathrm{T}, \mathrm{N}, \mathrm{M}$ status is undetermined, the MDT would advise patients to received further inspection such as endoscopic ultrasound, positron emission tomography-computed tomography (PET-CT) or magnetic resonance imaging (MRI) to determine the tumor invasion depth, perigastric nodal status and to further differentiate suspicious metastatic site. A second conference would be held after the suggested examinations were undertaken to determine the following strategy. In cases of patients refusing these examinations, the MDT will discuss the treatment plan based on the currently available information. Generally, the MDT recommend treatment plans based on the Chinese gastric cancer diagnosis and treatment guideline [11] (Published by the National Health Commission of the PRC) and the Japanese gastric cancer treatment guidelines[12]. For patients with controversial conditions, the MDT would try to develop personalized and integrated strategies for the patients, or recruit them for clinical studies. The expert panel would explain to patients the current state of the illness and the details of recommended treatment plan. With patients' consent, the doctors in charge will arrange further examination or treatment according to the recommendation.

\section{Propensity score matching}

To determine the impact of MDT, a propensity score matching method was used to select patients with similar baseline characteristics. To imitate randomized inclusion, matching factors included only preoperative parameters: gender, age, BMI, tumor location, differentiation, and clinical stage. The matching ratio was $1: 1$, the caliper was 0.03 . The matched pairs were divided into MDT group and non-MDT groups. Data analysis was based on the matched cohort.

\section{Data analysis}

Normally distributed continuous variables were expressed in the form of mean \pm standard deviation and were analyzed by Student's $t$-test. Categorical variables were analyzed by the chi-square test. The survival difference between the non-MDT and MDT group was compared using the Kaplan-Meier method, and the hazard ratios were calculated in the Cox regression model, a p-value $<0.05$ were identified as statistically significant. All statistical analyses were performed using SPSS software ver. 25.0 (IBM, Armonk, NY, USA) and R version 4.0 software (The R Foundation for Statistical Computing, Vienna, Austria; www.r-project.org).

\section{Results}

\section{Patient characteristics}

The process of patient enrollment and propensity score matching was shown in Figure 1. Within the 719 patients enrolled, a cohort of 378 patients was selected for analysis, including 189 in the Non-MDT group and 189 in the MDT group. The patient characteristics including baseline information were shown in Table 1 . As a result of propensity score matching, all baseline characteristics including gender, age, body mass index (BMI), tumor location and differentiation, and clinical stage were similar in these two groups. Patients were mostly male at a median age of 58 years with similar BMI. The majority of patients were diagnosed with poorly differentiated adenocarcinoma at clinical stage III. Also, the clinical stage was undetermined for one-fifth of the patients before surgery.

\section{Staging accuracy}

The accuracy of clinical staging in both groups was listed in Table 2. Patients that had received preoperative chemotherapy were not included in the staging accuracy analysis, since the final pathological stage could be vastly altered by chemotherapy. The clinical stages of patients in the MDT group were all reassessed and adjusted after MDT consultation. However, the data suggested that MDT consultation did not improve the clinical staging accuracy, as the accuracy of T (MDT 53\% vs Non-MDT 61\%, p=0.506), $\mathrm{N}$ (MDT 46.1\% vs Non-MDT 35.3\%, $\mathrm{p}=0.26$ ), and $\mathrm{M}$ stages (MDT 78.3\% vs Non-MDT 78.7\%, $\mathrm{p}=0.978$ ) was all statistically similar between these two groups. The only difference we observed was that patients in the MDT group tended to be over staged for the T stage (MDT 27\% vs Non-MDT 14.7\%, p=0.05).

\section{MDT recommendations and patient compliance}

As shown in Table 3, for all the patients at clinical stage I, MDT recommended direct surgical resection, 3 patients were recommended with endoscopic ultrasound to re-assure the $\mathrm{T}$ stage. For clinical stage II, most patients $(70 \%, 7 / 10)$ were recommended with direct surgical, 3 patients with bulky lymph nodes were recommended with neoadjuvant chemotherapy. For clinical stage III, the recommended strategies were divided, patients with adjacent organ invasion (T4a or T4b) or bulky lymph nodes tended to be recommended with neoadjuvant chemotherapy $(39 \%, 45 / 115)$, otherwise direct surgery were recommended $(42 \%, 48 / 115)$. For patients at stage IV, exploratory laparoscopy was recommended most $(57 \%, 8 / 14)$, chemotherapy $(43 \%$, 
$6 / 14)$ and palliative treatment $(43 \%, 6 / 14)$ was also frequently recommended. Patients with undetermined stage were mostly recommended with exploratory laparoscopy $(56 \%, 22 / 39)$, subsequent radical resection or chemotherapy shall be proceeded accordingly. Patients' compliance was clearly highest with direct surgical resection, laparoscopic explorations $(83 \%, 24 / 29)$ were accepted as a part of the resection surgery, no staging laparoscopic exploration without resection were accepted or performed. Only a minority of patients $(34 \%, 11 / 32)$ accepted further examination. None of them accepted the recommendation of radiotherapy or palliative therapy. These data indicated that patients' compliance could play a role in affecting the effectiveness of MDT consultation.

Table 1. Patient characteristics including the baseline information and $\mathrm{p}$-value of univariate analysis

\begin{tabular}{|c|c|c|c|c|}
\hline & $\begin{array}{l}\text { Non-MDT } \\
(\mathrm{n}=189)\end{array}$ & $\begin{array}{l}\text { MDT } \\
(n=189)\end{array}$ & Total $(\mathrm{n}=378)$ & $P$-value \\
\hline \multicolumn{5}{|l|}{ Gender } \\
\hline Female & $59(31.2 \%)$ & $60(31.7 \%)$ & $119(31.5 \%)$ & \multirow[t]{2}{*}{1} \\
\hline Male & $130(68.8 \%)$ & $129(68.3 \%)$ & $259(68.5 \%)$ & \\
\hline Age (years) & $58 \pm 12$ & $59 \pm 12$ & $58 \pm 12$ & 0.61 \\
\hline $\mathrm{BMI}\left(\mathrm{kg} / \mathrm{m}^{2}\right)$ & $21.7 \pm 3.6$ & $21.8 \pm 2.9$ & $21.7 \pm 3.3$ & 0.69 \\
\hline \multicolumn{5}{|l|}{ Tumor location } \\
\hline Esophagogastric junction & $15(7.9 \%)$ & $26(13.8 \%)$ & $41(10.8 \%)$ & \multirow[t]{5}{*}{0.1} \\
\hline Upper third & $38(20.1 \%)$ & $41(21.7 \%)$ & $79(20.9 \%)$ & \\
\hline Middle third & $47(24.9 \%)$ & $30(15.9 \%)$ & $77(20.4 \%)$ & \\
\hline Lower third & $88(46.6 \%)$ & $89(47.1 \%)$ & $177(46.8 \%)$ & \\
\hline Whole stomach & $1(0.5 \%)$ & $3(1.6 \%)$ & $4(1.1 \%)$ & \\
\hline \multicolumn{5}{|c|}{ Tumor histological classification } \\
\hline $\begin{array}{l}\text { Well differentiated } \\
\text { adenocarcinoma }\end{array}$ & $9(4.8 \%)$ & $7(3.7 \%)$ & $16(4.2 \%)$ & \multirow[t]{6}{*}{0.71} \\
\hline $\begin{array}{l}\text { Moderately } \\
\text { differentiated } \\
\text { adenocarcinoma }\end{array}$ & $51(27.0 \%)$ & $52(27.5 \%)$ & $103(27.2 \%)$ & \\
\hline $\begin{array}{l}\text { Poorly differentiated } \\
\text { adenocarcinoma }\end{array}$ & $116(61.4 \%)$ & $114(60.3 \%)$ & $230(60.8 \%)$ & \\
\hline Signet ring cell & $3(1.6 \%)$ & $8(4.2 \%)$ & $11(2.9 \%)$ & \\
\hline Mucous adenocarcinoma & $8(4.2 \%)$ & $7(3.7 \%)$ & $15(4.0 \%)$ & \\
\hline Papillary carcinoma & $2(1.1 \%)$ & $1(0.5 \%)$ & $3(0.8 \%)$ & \\
\hline \multicolumn{5}{|l|}{ Clinical stage } \\
\hline I & $15(7.9 \%)$ & $11(5.8 \%)$ & $26(6.9 \%)$ & \multirow[t]{5}{*}{0.32} \\
\hline II & $3(1.6 \%)$ & $10(5.3 \%)$ & $13(3.4 \%)$ & \\
\hline III & $116(61.4 \%)$ & $115(60.8 \%)$ & $231(61.1 \%)$ & \\
\hline IV & $12(6.3 \%)$ & $14(7.4 \%)$ & $26(6.9 \%)$ & \\
\hline Undetermined & $43(22.8 \%)$ & $39(20.6 \%)$ & $82(21.7 \%)$ & \\
\hline \multicolumn{5}{|c|}{ Preoperative chemotherapy } \\
\hline No & $136(72.0 \%)$ & $115(60.8 \%)$ & $251(66.4 \%)$ & \multirow[t]{2}{*}{0.03} \\
\hline Yes & $53(28.0 \%)$ & $74(39.2 \%)$ & $127(33.6 \%)$ & \\
\hline \multicolumn{5}{|c|}{ Preoperative chemotherapy regimen $(n=127)$} \\
\hline mFLOT & $31(58.5 \%)$ & $44(59.5 \%)$ & $75(59.1 \%)$ & \multirow[t]{3}{*}{0.45} \\
\hline SOX, FOLFOX or XELOX & $16(30.2 \%)$ & $26(35.1 \%)$ & $42(33.1 \%)$ & \\
\hline Other & $6(11.3 \%)$ & $4(5.4 \%)$ & $10(7.9 \%)$ & \\
\hline \multicolumn{5}{|l|}{ Resection approach } \\
\hline Open & $53(28.0 \%)$ & $33(17.5 \%)$ & $86(22.8 \%)$ & \multirow[t]{2}{*}{0.02} \\
\hline Laparoscopic & $136(72.0 \%)$ & $156(82.5 \%)$ & $292(77.2 \%)$ & \\
\hline \multicolumn{5}{|l|}{ Resection extend } \\
\hline Distal gastrectomy & $94(49.7 \%)$ & $95(50.3 \%)$ & $189(50.0 \%)$ & \multirow[t]{3}{*}{0.93} \\
\hline Proximal gastrectomy & $4(2.1 \%)$ & $5(2.6 \%)$ & $9(2.4 \%)$ & \\
\hline Total gastrectomy & $91(48.1 \%)$ & $89(47.1 \%)$ & $180(47.6 \%)$ & \\
\hline \multicolumn{5}{|l|}{ Completeness of resection } \\
\hline
\end{tabular}

\begin{tabular}{|c|c|c|c|c|}
\hline & $\begin{array}{l}\text { Non-MDT } \\
(\mathrm{n}=189)\end{array}$ & $\begin{array}{l}\text { MDT } \\
(n=189)\end{array}$ & Total $(n=378)$ & $P$-value \\
\hline R0 & $168(88.9 \%)$ & $177(93.7 \%)$ & $345(91.3 \%)$ & 0.73 \\
\hline $\mathrm{R} 1$ or R2 & $21(11.1 \%)$ & $12(6.3 \%)$ & $17(8.7 \%)$ & \\
\hline $\begin{array}{l}\text { Length of hospitalization } \\
\text { (days) }\end{array}$ & $14 \pm 9$ & $14 \pm 9$ & $14 \pm 9$ & 0.44 \\
\hline $\begin{array}{l}\text { Number of Harvested } \\
\text { lymph nodes }\end{array}$ & $32 \pm 15$ & $29 \pm 12$ & $31 \pm 14$ & 0.01 \\
\hline \multicolumn{5}{|l|}{ Vascular tumor embolus } \\
\hline No & $137(72.5 \%)$ & $132(69.8 \%)$ & $269(71.2 \%)$ & 0.65 \\
\hline Yes & $52(27.5 \%)$ & $57(30.2 \%)$ & $109(28.8 \%)$ & \\
\hline \multicolumn{5}{|l|}{ Nerve invasion } \\
\hline No & $112(59.3 \%)$ & $118(62.4 \%)$ & $230(60.8 \%)$ & 0.6 \\
\hline Yes & $77(40.7 \%)$ & $71(37.6 \%)$ & $148(39.2 \%)$ & \\
\hline Pathological stage & & & & 0.09 \\
\hline TONOMO & $8(4.8 \%)$ & $9(5.0 \%)$ & $17(4.9 \%)$ & \\
\hline IA & $14(8.4 \%)$ & $17(9.5 \%)$ & $31(9.0 \%)$ & \\
\hline IB & $10(6.0 \%)$ & $16(8.9 \%)$ & $26(7.5 \%)$ & \\
\hline IIA & $30(18.0 \%)$ & $45(25.1 \%)$ & $75(21.7 \%)$ & \\
\hline IIB & $46(27.5 \%)$ & $28(15.6 \%)$ & $74(21.4 \%)$ & \\
\hline IIIA & $21(12.6 \%)$ & $32(17.9 \%)$ & $53(15.3 \%)$ & \\
\hline IIIC & $20(12.0 \%)$ & $14(7.8 \%)$ & $34(9.8 \%)$ & \\
\hline IV & $13(7.8 \%)$ & $16(8.9 \%)$ & $29(8.4 \%)$ & \\
\hline Undetermined & $5(3.0 \%)$ & $2(1.1 \%)$ & $7(2.0 \%)$ & \\
\hline \multicolumn{5}{|c|}{$\begin{array}{l}\text { Abbreviations: MDT: Multidisciplinary team consultation; BMI: Body mass index; } \\
\mathrm{mFLOT:} \mathrm{Docetaxel} 50 \sim 60 \mathrm{mg} / \mathrm{m}^{2}+\text { Oxaliplatin } 85 \mathrm{mg} / \mathrm{m}^{2}+\text { Fluorouracil } 2800 \\
\mathrm{mg} / \mathrm{m}^{2} \text { iv over } 48 \text { hours; every } 2 \text { weeks. }\end{array}$} \\
\hline \multicolumn{5}{|c|}{$\begin{array}{l}\text { SOX: Oxaliplatin } 130 \mathrm{mg} / \mathrm{m}^{2} \text { iv + Tegafur Gimeracil Oteracil Potassium Capsule } \\
40 \sim 60 \mathrm{mg} \text { bid D1-D14; every } 3 \text { weeks. }\end{array}$} \\
\hline \multicolumn{5}{|c|}{$\begin{array}{l}\text { XELOX: Oxaliplatin } 130 \mathrm{mg} / \mathrm{m}^{2}+\text { Capecitabine } 1000 \mathrm{mg} / \mathrm{m}^{2} \text { bid D1-D14; every } 3 \\
\text { weeks. }\end{array}$} \\
\hline \multicolumn{5}{|c|}{$\begin{array}{l}\text { FOLFOX: Oxaliplatin } 85 \mathrm{mg} / \mathrm{m}^{2}+\text { Fluorouracil } 2800 \mathrm{mg} / \mathrm{m}^{2} \text { civ over } 48 \text { hours; } \\
\text { every } 2 \text { weeks. }\end{array}$} \\
\hline \multicolumn{5}{|c|}{$\begin{array}{l}\text { The dosage of the regimens listed above might be slightly modified according to } \\
\text { the preference of the oncologist. }\end{array}$} \\
\hline
\end{tabular}

\section{Treatments and clinical outcomes}

As shown in Table 1, more patients in the MDT group received preoperative chemotherapy than in the non-MDT group (39.2\% vs $28 \%, \mathrm{p}=0.03)$. More than half of the preoperative chemotherapy regimen was modified FLOT regimen (fluorouracil plus leucovorin, oxaliplatin, and docetaxel), which was similar in both groups. Other regimens were mainly platin-based doublet regimens, such as SOX, FOLFOX or XELOX. Laparoscopic surgery was the major resection approach in both groups but was more favored in the MDT group ( $82.5 \%$ vs $72 \%, p=0.02)$.

As for the surgical outcome, R0 resection was successfully achieved for around $91.3 \%$ of patients; however, no statistical difference was observed between the non-MDT group and the MDT group $(88.9 \%$ vs $93.7 \%, p=0.73)$. Also, the postoperative hospital stay days between these two groups were comparable (14 vs 14). Notably, 17 out of the 127 patients who received preoperative chemotherapy achieved a pathological complete response (13.4\%). The other pathological stages between these two groups were also similar. It should be noted that the MDT groups harvested fewer lymph nodes than the non-MDT group $(p=0.009)$. 
Table 2. Staging accuracy of the MDT group and the non-MDT group ( $n=278$, patients with neoadjuvant chemotherapy excluded)

\begin{tabular}{|c|c|c|c|c|c|c|c|c|c|c|c|c|}
\hline & & \multicolumn{7}{|c|}{ Pathological stage } & \multirow[t]{2}{*}{ Total } & \multirow[t]{2}{*}{ Correct } & \multirow[t]{2}{*}{ Overstage } & \multirow[t]{2}{*}{ Understage } \\
\hline & & pTis & $p T 1 a$ & $p T 1 b$ & $p T 2$ & $p T 3$ & $p T 4 a$ & $p T 4 b$ & & & & \\
\hline \multirow{19}{*}{ MDT } & $c T 1$ & - & 2 & 1 & - & 1 & - & - & 4 & $75 \%$ & NA & $25 \%$ \\
\hline & $c T 2$ & - & 4 & 4 & 3 & 4 & 1 & - & 16 & $18.8 \%$ & $50 \%$ & $31.3 \%$ \\
\hline & $c T 3$ & - & - & 3 & 15 & 48 & 3 & - & 69 & $69.6 \%$ & $26.1 \%$ & $4.3 \%$ \\
\hline & cT4a & - & - & 1 & - & 2 & 4 & - & 7 & $57.1 \%$ & $42.9 \%$ & NA \\
\hline & $c T 4 b$ & - & - & - & - & 2 & - & 3 & 5 & $60 \%$ & $40 \%$ & NA \\
\hline & $c T x$ & - & 3 & 1 & 1 & 6 & 3 & - & 14 & & & \\
\hline & Total & - & 9 & $1-$ & 19 & 63 & 11 & 3 & 115 & $53 \%$ & $27 \%$ & $7.8 \%$ \\
\hline & & $p N 0$ & $p N 1$ & $p N 2$ & $p N 3 a$ & $p N 3 b$ & & & & & & \\
\hline & cNO & 27 & 5 & 4 & 2 & 1 & & & 39 & $69.2 \%$ & NA & $30.8 \%$ \\
\hline & $c N 1$ & 9 & 8 & 8 & - & 1 & & & 26 & $30.8 \%$ & $34.6 \%$ & $34.6 \%$ \\
\hline & $c N 2$ & 5 & 2 & 9 & 2 & 1 & & & 19 & $47.4 \%$ & $36.8 \%$ & $15.8 \%$ \\
\hline & $c N 3$ & 1 & 2 & 1 & 3 & 6 & & & 13 & $69.2 \%$ & $30.8 \%$ & NA \\
\hline & $c N x$ & 7 & 4 & 1 & 2 & 4 & & & 18 & & & \\
\hline & Total & 49 & 21 & 23 & 9 & 13 & & & 115 & $46.1 \%$ & $17.4 \%$ & $20.9 \%$ \\
\hline & & $p M 0$ & $p M 1$ & $M x$ & & & & & & & & \\
\hline & $c M 0$ & 88 & 1 & - & & & & & 89 & $98.9 \%$ & NA & $1.1 \%$ \\
\hline & $c M 1$ & 3 & 2 & - & & & & & 5 & $40 \%$ & $60 \%$ & NA \\
\hline & $c M x$ & 19 & 2 & - & & & & & 21 & & & \\
\hline & Total & 110 & 5 & - & & & & & 115 & $78.3 \%$ & $2.6 \%$ & $0.9 \%$ \\
\hline \multirow[t]{20}{*}{ Non-MDT } & & $p$ Tis & $p T 1 a$ & $p T 1 b$ & $p T 2$ & $p T 3$ & $p T 4 a$ & $p T 4 b$ & & & & \\
\hline & $c T 1$ & 1 & 3 & 2 & - & - & - & - & 6 & $83.3 \%$ & $16.7 \%$ & NA \\
\hline & $c T 2$ & - & - & 3 & 5 & 4 & - & - & 12 & $41.7 \%$ & $25 \%$ & $33.3 \%$ \\
\hline & cT3 & - & 2 & 2 & 5 & 63 & 12 & 3 & 87 & $72.4 \%$ & $10.3 \%$ & $17.2 \%$ \\
\hline & cT4a & - & - & - & - & 6 & 9 & - & 15 & $60 \%$ & $40 \%$ & NA \\
\hline & $c T 4 b$ & - & - & - & - & - & 1 & 1 & 2 & $50 \%$ & $50 \%$ & NA \\
\hline & $c T x$ & - & 1 & 4 & - & 6 & 2 & 1 & 14 & & & \\
\hline & Total & 1 & 6 & 11 & 1- & 79 & 24 & 5 & 136 & $61 \%$ & $14.7 \%$ & $14 \%$ \\
\hline & & $p N 0$ & $p N 1$ & $p N 2$ & $p N 3 a$ & $p N 3 b$ & & & & & & \\
\hline & cNO & 20 & 11 & 10 & 3 & - & & & 44 & $45.5 \%$ & NA & $54.5 \%$ \\
\hline & $c N 1$ & 4 & 15 & 5 & 4 & 5 & & & 33 & $45.5 \%$ & $12.1 \%$ & $42.4 \%$ \\
\hline & $c N 2$ & 3 & 8 & 3 & 6 & 4 & & & 24 & $12.5 \%$ & $45.8 \%$ & $41.7 \%$ \\
\hline & $c N 3$ & - & 2 & 1 & 2 & 8 & & & 13 & $76.9 \%$ & $23.1 \%$ & NA \\
\hline & $c N x$ & 6 & 5 & 1 & 3 & 7 & & & 22 & & & \\
\hline & Total & 33 & 41 & $2-$ & 18 & 24 & & & 136 & $35.3 \%$ & $13.2 \%$ & $35.3 \%$ \\
\hline & & $p M 0$ & $p M 1$ & $M x$ & & & & & & & & \\
\hline & $c M 0$ & 104 & 2 & - & & & & & 106 & $98.1 \%$ & NA & $1.9 \%$ \\
\hline & $c M 1$ & 3 & 3 & - & & & & & 6 & $50 \%$ & $50 \%$ & NA \\
\hline & $c M x$ & 18 & 1 & 5 & & & & & 24 & & & \\
\hline & Total & 125 & 6 & 5 & & & & & 136 & $78.7 \%$ & $2.2 \%$ & $1.5 \%$ \\
\hline
\end{tabular}

Table 3. Recommendations of the MDT and the corresponding patient compliance

\begin{tabular}{|c|c|c|c|c|c|c|}
\hline Clinical stage & $\begin{array}{l}\text { Pre-operative } \\
\text { chemotherapy }\end{array}$ & Direct tumor resection & Further investigation & Exploratory laparoscopy & Radiotherapy & Palliative therapy \\
\hline $\mathrm{I}(\mathrm{n}=11)$ & 0 & $11(100 \%)$ & $4(36 \%)$ & 0 & 0 & 0 \\
\hline II $(n=10)$ & $3(30 \%)$ & $7(70 \%)$ & $1(10 \%)$ & $1(10 \%)$ & $1(10 \%)$ & 0 \\
\hline III (n=115) & $45(39 \%)$ & $48(42 \%)$ & $19(17 \%)$ & $13(11 \%)$ & $4(3 \%)$ & 0 \\
\hline IV $(n=14)$ & $6(43 \%)$ & $2(14 \%)$ & $2(14 \%)$ & $8(57 \%)$ & $3(21 \%)$ & $6(43 \%)$ \\
\hline Undetermined (n=39) & $14(36 \%)$ & $1(3 \%)$ & $6(15 \%)$ & $22(56 \%)$ & $3(8 \%)$ & $1(3 \%)$ \\
\hline Total $(\mathrm{n}=189)$ & $68(36 \%)$ & $84(44 \%)$ & $32(17 \%)$ & $29(15 \%)$ & $11(6 \%)$ & $7(4 \%)$ \\
\hline Compliance & $44 / 68,65 \%$ & $68 / 84,81 \%$ & $11 / 32,34 \%$ & $24 / 29,83 \%$ & 0 & 0 \\
\hline
\end{tabular}

\section{Survival analysis}

Median follow-up time for the cohort was $17 \pm 11$ months. The 1-year and 3-year overall survival rate for the total cohort was $92 \%$ and $66 \%, 90 \%, 62 \%$ for the non-MDT group, 93\%, 68\% for the MDT group. As shown in Figure 2, the overall survival was comparable between the MDT and non-MDT groups $(p=0.54)$. In subgroup survival analysis (Figure 3 ), MDT groups also failed to show any superiority compared to the non-MDT group. Therefore, these data suggested that MDT consultation has limited effects on improving the survival of the patients.

\section{Discussion}

Although the MDT approach has been proposed in cancer treatments, especially for individualized therapies, its effectiveness remains controversial due to the lack of strong evidence demonstrating its advantages in improving clinical outcomes [13, 14]. This single-center retrospective study suggested that MDT consultation has limited effectiveness in improving staging accuracy and treatment outcomes including overall survival for patients with resectable gastric cancer. Moreover, the data indicated that patient compliance may affect the effectiveness of MDT consultation. 


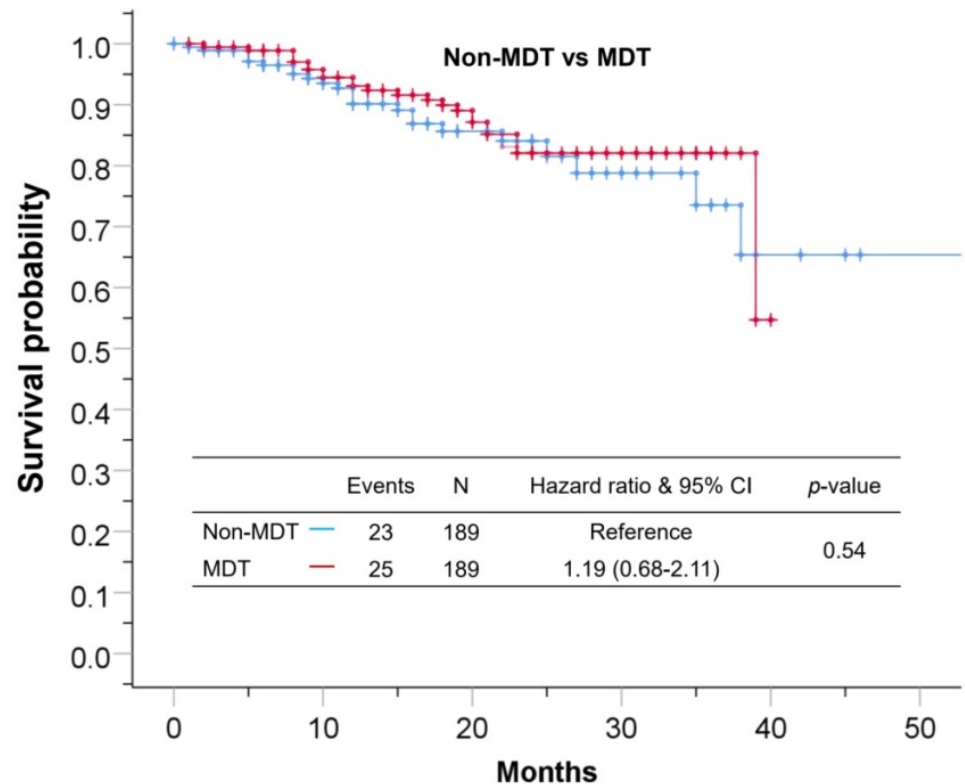

Figure 2. The Kaplan-Meier curve showing no statistical difference between patients in the MDT group and patients in the non-MDT group.

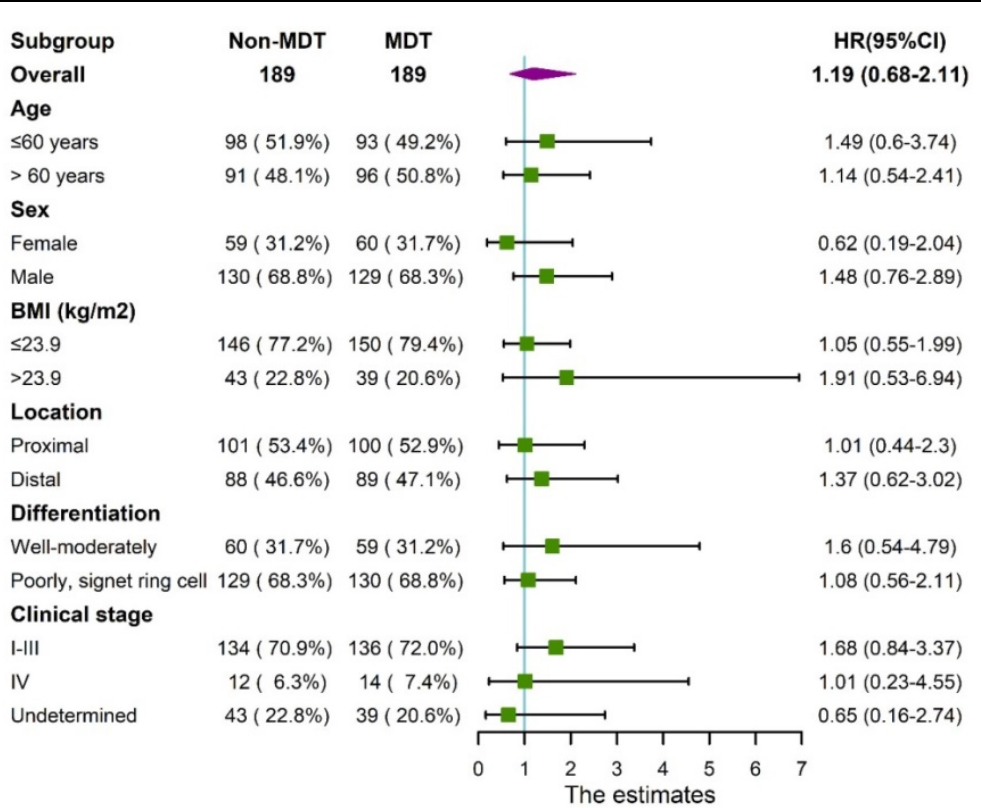

Figure 3. Forest plot showing no significant benefit from MDT consultation for patients in different subgroups.

One important purpose of MDT is to improve staging accuracy of cancer patients. However, contrary to the previous study [8], our results showed that the accuracy of TNM staging was not improved by MDT consultation even after excluding patients who received preoperative chemotherapy that may ultimately alter the final pathological stage of tumors. One possible reason is the poor patient compliance that leads to insufficient examinations. To improve staging accuracy, multi-modality imaging techniques must be applied. For example, endoscopic ultrasonography has been proven to be the most reliable and accurate technique for T staging [15-17],
PET-CT and MRI are important approaches for differentiating suspicious metastatic site [18-21]. In our study cohort, these examinations were frequently recommended by the MDT but rarely accepted by the patients due to the worry of further delay of surgery and expensive cost.

Regarding peritoneum metastasis, staging laparoscopy has been recommended by several studies [22-24]. Although our data showed that the recommendation of exploratory laparoscopy was accepted by the majority of patients, it was only accepted as part of the laparoscopic gastrectomy surgery; and yet none of the exploratory laparoscopies were performed simply as a method of staging inspection without immediate subsequent resection. Thus, the clinical stages of the MDT group were still mostly established on routine contrast-enhanced CT imaging, which limited the room for improvement, even with the reassessment by the MDT.

As for the treatment plan, all patients at the early stage (clinical stage I) were recommended with direct resection surgery by the MDT. Endoscopic submucosal dissection was only applicable for patients with tumor invasion depth under T1a and with no evidence of lymph node metastasis [25]. Also, the probability of insidious lymph node metastasis in early gastric cancer is not negligible [26]. Hence, endoscopic resection was rarely recommended. For patients at clinical stage II-III, there was a controversy on the necessity of neoadjuvant chemotherapy. The Chinese gastric cancer diagnosis and treatment guideline recommended it only for cT3/4N+ patients[11], but it also stated that the survival benefit was still uncertain and well-designed trials were lacking [27, 28], the guideline encouraged MDT to develop individualized strategy based on the tumor feature and the patients' condition. The Japanese guideline recommend neoadjuvant chemotherapy for cT2-4 patients with evident of bulky lymph nodes metastasis [29]. Thus, our MDT had tried to balance the differences across guidelines and attempt to develop more personalized treatment strategies for this subgroup. Overall speaking, the MDT tends to recommend neoadjuvant chemotherapy to patients with good performance status, present of adjacent organ invasion or bulky lymph node, and direct surgical resection otherwise. As a result, patients in 
the MDT group had received more preoperative chemotherapy, applied regimens mainly include mFLOT [28] and SOX [30], but the achievement of R0 resection and survival was not improved. For patients at clinical stage IV, the treatment recommendations were vastly different due to the great heterogeneous nature of the disease. Surgery could only be attempted when curative resection is achievable or in case of obstruction or bleeding [29], but in most case, systemic chemotherapy was the first choice. In this study, all patients enrolled had already received gastrectomy. But preoperative MDT consultation failed to show any positive impact on the survival of this subgroup. The MDT had also recommended a variety of treatments and supportive care that may have been beneficial to these patients [31], but regrettably, they were rarely accepted by the patients. As for radiotherapy [32], the National Comprehensive Cancer Network guideline for gastric cancer recommends it as part of perioperative therapy, but in the guidelines of Asia, it is recommended only in patients with unresectable metastatic sites [29, 33]. In our study, less than $10 \%$ of patients were recommended to receive radiotherapy, but none of them complied.

Regarding survival benefits, MDT consultation did not improve overall survival rate in our study. In the subgroup analysis, the MDT group did not show any survival advantage for patients with tumors in different locations, differentiation, or different clinical stages, as shown in the forest plot in Figure 3. Possible explanations could be as followed. First, the patients' poor compliance with further examinations weakened the effectiveness of MDT consultation. Moreover, most of treatment plans recommended for patients at advanced stages were invalidated strategies, such as neoadjuvant chemotherapy and radiotherapy, which were controversial in term of their survival benefit. Besides, the recommended approaches by the MDT for patients at early stages were mostly standardized plans, which was not much different from the non-MDT group. These factors could notably limit the room for survival improvement of MDT consultation. Thus, we believe that patient compliance and staging accuracy are key factors affecting the survival of patients with gastric cancer.

Noticeably, several limitations to this study need to be acknowledged. First, selection bias cannot be neglected. All patients enrolled in this study eventually underwent gastrectomy, those who refused surgery or lost the opportunity to undergone surgery due to progression during chemotherapy were not included, which may result in selection bias. Second, the number of studied populations distributed unevenly among the various stages. In particular, most of the enrolled patients were at stage III; and the sample size of stage I, II, and IV may not be sufficient to testify the effectiveness of MDT. Third, the follow-up time for this cohort was relatively short, which could weaken the statistical significance of survival analysis. Despite these limitations, this is the first study exploring the effectiveness of MDT consultation on patients with resectable gastric cancer with detailed data provided. The utility of propensity score matching method allows us to imitate randomized inclusion and compare the effect of MDT between the two groups, making the results more persuasive.

\section{Conclusion}

This study demonstrates that MDT consultation has limited effect in improving the staging accuracy and treatment outcomes including the survival benefit for patients with resectable gastric cancer, likely due to insufficient examination and poor patient compliance. To improve the effectiveness of MDT consultation, further efforts should be made to improve staging accuracy and the compliance of patients.

\section{Acknowledgments}

The authors acknowledge and thank all research participants who supported and facilitated this research.

\section{Funding}

This study is supported by the Guangzhou Science and Technology Project (grant number 201803010040) and Nation Key Clinical Discipline.

\section{Ethics approval}

The study was reviewed and approved by the ethics committee of The Sixth Affiliated Hospital, Sun Yat-Sen University. This study was conducted in accordance with the 1964 Helsinki Declaration.

\section{Consent to participate}

All study participants, or their legal guardians, were provided with informed consent prior to study by the follow-up office.

\section{Consent for publication}

All authors have approved the manuscript and have agreed with its submission to the Journal of Cancer Research and Clinical Oncology for publication.

\section{Availability of data and material}

Data queries can be emailed to Dr. Xiaochun Meng (mengxch3@mail.sysu.edu.cn). 


\section{Authors' contributions}

Peng JS and Meng XC designed the study; Chen $\mathrm{YH}$, Xiang J, and Liu D contributed equally in acquiring, analyzing, interpreting the data, and drafted the initial manuscript. Xiao J, Xiong F, Wei KK, Liu AH, Chen S, Zhu YX, Meng XC and Peng JS made important revisions to the manuscript. Yonghe Chen, Jun Xiang and Dan Liu contributed equally to this work.

\section{Authors ORCID}

Yonghe Chen: 0000-0001-5957-3872; Jun Xiang: 0000-0002-9264-4182; Dan Liu: 0000-0002-8914-6203; Jian Xiao: 0000-0001-8224-4874; Kaikai Wei: 0000-00016632-0822; Xiaochun Meng: 0000-0002-1302-0380; Junsheng Peng: 0000-0002-7975-4176.

\section{Competing Interests}

The authors have declared that no competing interest exists.

\section{References}

1. Bray F, Ferlay J, Soerjomataram I, et al. Global cancer statistics 2018: GLOBOCAN estimates of incidence and mortality worldwide for 36 cancers in 185 countries. CA: a cancer journal for clinicians. 2018; 68: 394-424

2. Bouvier AM, Sant M, Verdecchia A, et al. What reasons lie behind long-term survival differences for gastric cancer within Europe? European journal of cancer (Oxford, England : 1990). 2010; 46: 1086-92.

3. Davis PA, Sano T. The difference in gastric cancer between Japan, USA and Europe: what are the facts? what are the suggestions? Critical reviews in oncology/hematology. 2001; 40: 77-94

4. Van Laethem JL, Carneiro F, Ducreux M, et al. The multidisciplinary management of gastro-oesophageal junction tumours: European Society of Digestive Oncology (ESDO): Expert discussion and report from the 16th ESMO World Congress on Gastrointestinal Cancer, Barcelona. Digestive and liver disease : official journal of the Italian Society of Gastroenterology and the Italian Association for the Study of the Liver. 2016; 48: 1283-9.

5. Silbermann M, Pitsillides B, Al-Alfi N, et al. Multidisciplinary care team for cancer patients and its implementation in several Middle Eastern countries. Annals of oncology: official journal of the European Society for Medical Oncology. 2013; 24 Suppl 7: vii41-7.

6. Soukup T, Lamb BW, Arora S, et al. Successful strategies in implementing a multidisciplinary team working in the care of patients with cancer: an overview and synthesis of the available literature. Journal of multidisciplinary healthcare. 2018; 11: 49-61.

7. Wagner AD, Lordick F, Grabsch HI, et al. Multidisciplinary management of stage II-III gastric and gastro-oesophageal junction cancer. European journal of cancer (Oxford, England: 1990). 2020; 124: 67-76.

8. Davies AR, Deans DA, Penman I, et al. The multidisciplinary team meeting improves staging accuracy and treatment selection for gastro-esophageal cancer. Diseases of the esophagus: official journal of the International Society for Diseases of the Esophagus. 2006; 19: 496-503.

9. Johnston FM, Beckman M. Updates on Management of Gastric Cancer. Current oncology reports. 2019; 21: 67

10. Amin MB ES, Greene F, Byrd DR, Brookland RK, Washington MK Gershenwald JE, Compton CC, Hess KR, et al. AJCC Cancer Staging Manual. 8th ed: Springer International Publishing: American Joint Commission on Cancer; 2017.

11. National Health Commission of the People Republic of China. Gastric Cancer Diagnosis and Treatment Guideline 2011 ed; 2011.

12. Japanese Gastric Cancer A. Japanese gastric cancer treatment guidelines 2014 (ver. 4). Gastric cancer : official journal of the International Gastric Cancer Association and the Japanese Gastric Cancer Association. 2017; 20: 1-19.

13. Fleissig A, Jenkins V, Catt $\mathrm{S}$, et al. Multidisciplinary teams in cancer care: are they effective in the UK? The Lancet Oncology. 2006; 7: 935-43.

14. Taylor C, Munro AJ, Glynne-Jones R, et al. Multidisciplinary team working in cancer: what is the evidence? BMJ (Clinical research ed). 2010; 340: c951.

15. Fairweather M, Jajoo K, Sainani N, et al. Accuracy of EUS and CT imaging in preoperative gastric cancer staging. Journal of surgical oncology. 2015; 111: 1016-20

16. Kim J, Kim SG, Chung $\mathrm{H}$, et al. Clinical efficacy of endoscopic ultrasonography for decision of treatment strategy of gastric cancer. Surgical endoscopy. 2018; 32: 3789-97.
17. Takamaru H, Yoshinaga S, Takisawa $\mathrm{H}$, et al. Endoscopic Ultrasonography Miniature Probe Performance for Depth Diagnosis of Early Gastric Cancer with Suspected Submucosal Invasion. Gut and liver. 2019.

18. Borggreve AS, Goense L, Brenkman HJF, et al. Imaging strategies in the management of gastric cancer: current role and future potential of MRI. The British journal of radiology. 2019; 92: 20181044.

19. Liu Y, Zheng D, Liu JJ, et al. Comparing PET/MRI with PET/CT for Pretreatment Staging of Gastric Cancer. Gastroenterology research and practice. 2019; 2019: 9564627.

20. Tang L, Wang XI, Baba $\mathrm{H}$, et al. Gastric cancer and image-derived quantitative parameters: Part 2-a critical review of DCE-MRI and (18)F-FDG PET/CT findings. European radiology. 2020; 30: 247-60.

21. Findlay JM, Antonowicz S, Segaran A, et al. Routinely staging gastric cancer with (18)F-FDG PET-CT detects additional metastases and predicts early recurrence and death after surgery. European radiology. 2019; 29: 2490-8.

22. Fukagawa T. Role of staging laparoscopy for gastric cancer patients. Annals of gastroenterological surgery. 2019; 3: 496-505.

23. Brenkman HJF, Gertsen EC, Vegt E, et al. Evaluation of PET and laparoscopy in STagIng advanced gastric cancer: a multicenter prospective study (PLASTIC-study). BMC cancer. 2018; 18: 450.

24. Machairas N, Charalampoudis P, Molmenti EP, et al. The value of staging laparoscopy in gastric cancer. Annals of gastroenterology. 2017; 30: 287-94.

25. Ono H, Yao K, Fujishiro $M$, et al. Guidelines for endoscopic submucosal dissection and endoscopic mucosal resection for early gastric cancer. Digestive endoscopy: official journal of the Japan Gastroenterological Endoscopy Society. 2016; 28: 3-15.

26. Hölscher AH, Drebber U, Mönig SP, et al. Early gastric cancer: lymph node metastasis starts with deep mucosal infiltration. Annals of surgery. 2009; 250: 791-7.

27. Reddavid R, Sofia S, Chiaro P, et al. Neoadjuvant chemotherapy for gastric cancer. Is it a must or a fake? World journal of gastroenterology. 2018; 24: 274-89.

28. Al-Batran SE, Homann N, Pauligk C, et al. Perioperative chemotherapy with fluorouracil plus leucovorin, oxaliplatin, and docetaxel versus fluorouracil or capecitabine plus cisplatin and epirubicin for locally advanced, resectable gastric or gastro-oesophageal junction adenocarcinoma (FLOT4): a randomised, phase 2/3 trial. Lancet (London, England). 2019; 393: 1948-57.

29. Japanese Gastric Cancer A. Japanese gastric cancer treatment guidelines 2018 (5th edition). Gastric cancer : official journal of the International Gastric Cancer Association and the Japanese Gastric Cancer Association. 2020.

30. Xue K, Ying X, Bu Z, et al. Oxaliplatin plus S-1 or capecitabine as neoadjuvant or adjuvant chemotherapy for locally advanced gastric cancer with D2 lymphadenectomy: 5-year follow-up results of a phase II-III randomized trial. Chinese journal of cancer research $=$ Chung-kuo yen cheng yen chiu. 2018; 30: 516-25.

31. Beom SH, Choi YY, Baek SE, et al. Multidisciplinary treatment for patients with stage IV gastric cancer: the role of conversion surgery following chemotherapy. BMC cancer. 2018; 18: 1116

32. Hallissey MT, Dunn JA, Ward LC, et al. The second British Stomach Cancer Group trial of adjuvant radiotherapy or chemotherapy in resectable gastric cancer: five-year follow-up. Lancet (London, England). 1994; 343: 1309-12.

33. Wang FH, Shen L, Li J, et al. The Chinese Society of Clinical Oncology (CSCO): clinical guidelines for the diagnosis and treatment of gastric cancer. Cancer communications (London, England). 2019; 39: 10. 